\title{
RECURRENCE FORM FOR DETERMINANT OF A HEPTADIAGONAL SYMMETRIC TOEPLITZ MATRIX
}

\author{
Jolanta Borowska, Lena Lacińska \\ Institute of Mathematics, Czestochowa University of Technology \\ Częstochowa, Poland \\ jolanta.borowska@im.pcz.pl,lena.lacinska@im.pcz.pl
}

\begin{abstract}
In this paper we show that the determinant of heptadiagonal symmetric Toeplitz matrix can be represented by a particular solution of the system of three homogeneous linear recurrence equations. The general considerations are illustrated by certain numerical example implementated in the Maple system.
\end{abstract}

Keywords: Toeplitz matrix, heptadiagonal matrix, determinant, linear recurrence equations

\section{Introduction}

The subject of consideration is a heptadiagonal symmetric Toeplitz matrix of the form

$$
\mathbf{A}_{n}=\left[\begin{array}{ccccccccccc}
a & b & c & d & & & & & & & \\
b & a & b & c & d & & & & & & \\
c & b & a & b & c & d & & & & & \\
d & c & b & a & b & c & d & & & & \\
& \ddots & \ddots & \ddots & \ddots & \ddots & \ddots & \ddots & & & \\
& & \ddots & \ddots & \ddots & \ddots & \ddots & \ddots & \ddots & & \\
& & & d & c & b & a & b & c & d & \\
& & & & d & c & b & a & b & c & d \\
& & & & & d & c & b & a & b & c \\
& & & & & & d & c & b & a & b \\
& & & & & & & d & c & b & a
\end{array}\right]
$$

It means that $\mathbf{A}_{n}=\left[a_{i j}\right]_{n \times n}$, where

$$
a_{i j}= \begin{cases}a, & |i-j|=0 \\ b, & |i-j|=1 \\ c, & |i-j|=2 \\ d, & |i-j|=3 \\ 0, & \text { otherwise }\end{cases}
$$


Heptadiagonal Toeplitz matrices are a special class of band Toeplitz matrices which play an important role in physics, mathematics, statistics, and signal processing. The numerical algorithms for computing determinants or eigenvalues of heptadiagonal Toeplitz matrices are proposed for example in [1-3]. In the present paper we are to show that the determinant of heptadiagonal symmetric Toeplitz matrix can be described by a system of three linear recurrence equations. This approach enables us to construct a simple and effective numerical algorithm for calculating determinant of considered matrix with fixed dimension and fixed elements on bands.

\section{The main results}

In this section we present a recurrence relation for determinant of matrix (1). To this end we introduce two auxiliary heptadiagonal matrices $\overline{\mathbf{A}}_{n}$ and $\hat{\mathbf{A}}_{n}$ of the form

$$
\overline{\mathbf{A}}_{n}=\left[\begin{array}{ccccccccccc}
a & b & c & d & & & & & & & \\
b & a & b & c & d & & & & & & \\
c & b & a & b & c & d & & & & & \\
d & c & b & a & b & c & d & & & & \\
& \ddots & \ddots & \ddots & \ddots & \ddots & \ddots & \ddots & & & \\
& & \ddots & \ddots & \ddots & \ddots & \ddots & \ddots & \ddots & & \\
& & & d & c & b & a & b & c & d & \\
& & & & d & c & b & a & b & c & d \\
& & & & & d & c & b & a & b & c \\
& & & & & & d & c & b & a & b \\
& & & & & & & 0 & d & c & b
\end{array}\right]
$$

$$
\hat{\mathbf{A}}_{n}=\left[\begin{array}{ccccccccccc}
a & b & c & d & & & & & & & \\
b & a & b & c & d & & & & & & \\
c & b & a & b & c & d & & & & & \\
d & c & b & a & b & c & d & & & & \\
& \ddots & \ddots & \ddots & \ddots & \ddots & \ddots & \ddots & & & \\
& & \ddots & \ddots & \ddots & \ddots & \ddots & \ddots & \ddots & & \\
& & & d & c & b & a & b & c & d & \\
& & & & d & c & b & a & b & c & 0 \\
& & & & & d & c & b & a & b & d \\
& & & & & & d & c & b & a & c \\
& & & & & & & 0 & d & c & a
\end{array}\right]
$$


It means that $\overline{\mathbf{A}}_{n}=\left[b_{i j}\right]_{n \times n}, \hat{\mathbf{A}}_{n}=\left[c_{i j}\right]_{n \times n}$ where

$$
b_{i j}=\left\{\begin{array}{ll}
b, & i=n, j=n \\
c, & i=n, j=n-1 \\
d, & i=n, j=n-2, c_{i j} \\
0, & i=n, j=n-3 \\
a_{i j}, & \text { otherwise }
\end{array}= \begin{cases}a, & i=n, j=n \\
c, & i=n-1, j=n \\
d, & i=n-2, j=n \\
0, & i=n-3, j=n \\
c, & i=n, j=n-1 \\
d, & i=n, j=n-2 \\
0, & i=n, j=n-3 \\
a_{i j} & \text { otherwise }\end{cases}\right.
$$

By $W_{n}, \bar{W}_{n}, \hat{W}_{n}$ we denote determinants of matrices $\mathbf{A}_{n}, \overline{\mathbf{A}}_{n}, \hat{\mathbf{A}}_{n}$, respectively. In order to obtain the determinant $W_{n}$ we adapt the approach proposed in [4]. After repeated use of the theorem of Laplace expansion we finally obtain a system of three linear recurrence equations

$$
\left\{\begin{aligned}
W_{n+7}= & a W_{n+6}+b d\left(b d-2 c^{2}\right) W_{n+3}+d^{2}\left(2 c^{3}-4 b c d+b^{2} c+a d^{2}\right) W_{n+2}+ \\
& +d^{3}\left(2 c^{2} d+b^{2} d-b c^{2}-d^{3}\right) W_{n+1}-b c d^{5} W_{n}-b \bar{W}_{n+6}+b c \bar{W}_{n+5}+ \\
& +d\left(2 a c-b^{2}\right) \bar{W}_{n+4}+b d^{2}(2 c-a) \bar{W}_{n+3}+d^{3}\left(2 b d-b^{2}-2 c^{2}\right) \bar{W}_{n+2}+ \\
& +c d^{4}(b-2 d) \bar{W}_{n+1}+b d^{6} \bar{W}_{n}-c^{2} \hat{W}_{n+5}+d(b c-d a) \hat{W}_{n+4} \\
\bar{W}_{n+6}= & b W_{n+5}-b c d^{2} W_{n+2}+d^{3}\left(c^{2}-b d\right) W_{n+1}+d^{5} c W_{n}-c \bar{W}_{n+5}+ \\
& +b d \bar{W}_{n+4}+a d^{2} \bar{W}_{n+3}+b d^{3} \bar{W}_{n+2}-c d^{4} \bar{W}_{n+1}-d^{6} \bar{W}_{n}-c d \hat{W}_{n+4} \\
\hat{W}_{n+2}= & a W_{n+1}-c^{2} W_{n}+2 c d \bar{W}_{n}-d^{2} \hat{W}_{n}
\end{aligned}\right.
$$

where $n \in \mathbf{N}$.

In order to obtain value of determinant $W_{n}$ we must take into account the system of equations (4) together with initial conditions of the form

$$
\begin{aligned}
W_{1} & =a, \quad W_{2}=a^{2}-b^{2}, \quad W_{3}=a W_{2}-b \bar{W}_{2}+b^{2} c-a c^{2} \\
W_{4} & =a W_{3}-b \bar{W}_{3}+b c \bar{W}_{2}-c^{2} \hat{W}_{2}+a d(3 b c-a d)+b d\left(b d-b^{2}-2 c^{2}\right) \\
W_{5} & =a W_{4}-b \bar{W}_{4}+b c \bar{W}_{3}+d\left(2 a c-b^{2}\right) \bar{W}_{2}-c^{2} \hat{W}_{3}+d(b c-a d) \hat{W}_{2}+ \\
& +b c d\left(3 b d-2 a c-4 d^{2}\right)+d^{2}\left(2 c^{3}+a d^{2}\right)
\end{aligned}
$$




$$
\begin{aligned}
W_{6} & =a W_{5}+b d\left(b d-2 c^{2}\right) W_{2}-b \bar{W}_{5}+b c \bar{W}_{4}+d\left(2 a c-b^{2}\right) \bar{W}_{3}+ \\
& +b d^{2}(2 c-a) \bar{W}_{2}-c^{2} \hat{W}_{4}+d(b c-a d) \hat{W}_{3}+c^{2} d^{2}(2 a c-3 b d)+ \\
& +a b c d\left(b d-4 d^{2}\right)+d^{4}\left(a^{2}+2 c^{2}+3 b^{2}-d^{2}\right)-d^{3} b^{3} \\
W_{7} & =a W_{6}+b d\left(b d-2 c^{2}\right) W_{3}+d^{2}\left(2 c^{3}-4 b c d+b^{2} c+a d^{2}\right) W_{2}-b \bar{W}_{6}+b c \bar{W}_{5}+ \\
& +d\left(2 a c-b^{2}\right) \bar{W}_{4}+b d^{2}(2 c-a) \bar{W}_{3}+d^{3}\left(2 b d-b^{2}-2 c^{2}\right) \bar{W}_{2}-c^{2} \hat{W}_{5}+ \\
& +d(b c-a d) \hat{W}_{4}+a d^{3}\left(2 c^{2} d+b^{2} d-b c^{2}-d^{3}\right)+b c d^{4}(b-3 d) \\
\overline{W_{1}} & =b, \quad \overline{W_{2}}=a b-b c, \quad \bar{W}_{3}=b W_{2}-c \bar{W}_{2}+d\left(b^{2}-a c\right) \\
\bar{W}_{4} & =b W_{3}-c \bar{W}_{3}+b d \bar{W}_{2}-c d \hat{W}_{2}+b d^{2}(a-c) \\
\bar{W}_{5} & =b W_{4}-c \bar{W}_{4}+b d \bar{W}_{3}+a d^{2} \bar{W}_{2}-c d \hat{W}_{3}+d^{3}\left(b^{2}+c^{2}\right)-b d^{2}\left(d^{2}+a c\right) \\
\bar{W}_{6} & =b W_{5}-b c d^{2} W_{2}-c \bar{W}_{5}+b d \bar{W}_{4}+a d^{2} \bar{W}_{3}+b d^{3} \bar{W}_{2}+ \\
& -c d \hat{W}_{4}+c d^{3}\left(d^{2}+a c\right)-b d^{4}(a+c) \\
\hat{W}_{1} & =a, \quad \hat{W}_{2}=a^{2}-c^{2}
\end{aligned}
$$

The above initial conditions are obtained by calculation of pertinent determinants. Hence the value of determinant of heptadiagonal matrix (1) is the particular solution of the system of equations (4) fulfilling the initial conditions (5). It can be observed that the direct solution of the system of equations (4) can be obtained only in some very special cases. However, for an arbitrary but fixed $n \in \mathbf{N}$ the proposed system of equations is suitable for constructing an effective algorithm for computation of determinant $W_{n}$.

\section{Remark 1.}

If $d=0$ then we deal with pentadiagonal symmetric Toeplitz matrix of the form

$$
\mathbf{A}_{n}=\left[\begin{array}{ccccccc}
a & b & c & & & & \\
b & a & b & c & & & \\
c & b & a & b & c & & \\
& \ddots & \ddots & \ddots & \ddots & \ddots & \\
& & \ddots & \ddots & \ddots & \ddots & c \\
& & & c & b & a & b \\
& & & & c & b & a
\end{array}\right]_{n \times n}
$$


It can be seen that in this case the system of equations (4) can be reduced to a system of two recurrence equations

$$
\left\{\begin{array}{l}
W_{n+4}=a W_{n+3}-a c^{2} W_{n+1}+c^{4} W_{n}-b \bar{W}_{n+3}+b c \bar{W}_{n+2} \\
\bar{W}_{n+1}=b W_{n}-c \bar{W}_{n}
\end{array}\right.
$$

where $n \in \mathbf{N}$.

At the same time initial conditions reduce to the form

$$
\left\{\begin{array}{l}
W_{1}=a \\
W_{2}=a^{2}-b^{2} \\
W_{3}=a W_{2}-b \bar{W}_{2}+b^{2} c-a c^{2} \\
W_{4}=a W_{3}-b^{2} W_{2}+2 b c \bar{W}_{2}+c^{4}-a^{2} c^{2} \\
\overline{W_{1}}=b
\end{array}\right.
$$

Hence the determinant of matrix (6) is the particular solution of the system of equations (7) fulfilling the initial conditions (8), compare [4].

\section{Remark 2.}

If $c=d=0$ then we deal with tridiagonal symmetric Toeplitz matrix of the form

$$
\mathbf{A}_{n}=\left[\begin{array}{ccccccc}
a & b & & & & & \\
b & a & b & & & & \\
& b & a & b & & & \\
& & b & a & b & & \\
& & & \ddots & \ddots & \ddots & \\
& & & & b & a & b \\
& & & & & b & a
\end{array}\right]_{n \times n}
$$

In this case from (4) we obtain finally one recurrence equation describing the determinant of matrix (9)

$$
W_{n+2}=a W_{n+1}-b^{2} W_{n}
$$

where $n \in \mathbf{N}$.

At the same time initial conditions reduce to the form

$$
W_{1}=a, W_{2}=a^{2}-b^{2}
$$

Hence the determinant of tridiagonal matrix (9) is the particular solution of the equation (10) fulfilling the initial conditions (11). 


\section{Illustrative example}

In order to illustrate the general results obtained in the previous section we consider a special form of heptadiagonal matrix (1) in which $a=1, b=2, c=3, d=4$. Moreover, assuming that matrix under considerations has the order $10^{5} \times 10^{5}$. Bearing in mind the above assumptions and formula (4) determinant of this matrix is given by the system of three linear recurrence equations with constant coefficients. It can be observed that it is impossible to solve such a system of recurrence equations using known analytical methods, [5]. Therefore, in order to calculate determinant of matrix under considerations, we construct an algorithm which will be implemented in the Maple algebra system. In order to speed up Maple calculations, the formula for $\hat{W}_{n}$ in (4) is transformed to the form

$$
\hat{W}_{n+5}=a W_{n+4}-c^{2} W_{n+3}+2 c d \bar{W}_{n+3}-d^{2} \hat{W}_{n+3}
$$

and the additional initial conditions for $\hat{W}_{3}, \hat{W}_{4}$ are imposed. Moreover let us denote $F=\bar{W}, G=\hat{W}$. Bearing in mind (4) and the above assumptions we apply the following syntax in Maple:

Step 1 (generating of initial conditions)

with(LinearAlgebra):

$h(i, j):=$ piecewise $(i=j, a, i=j+1, b, i=j-1, b, i=j+2, c, i=j-2, c, i=j+3, d, i=j-3, d)$ :

$p(i, j):=$ piecewise $(i=n$ and $j=n, b, i=n$ and $j=n-1, c, i=n$ and $j=n-2, d, i=n$ and $j=n-3,0, h(i, j))$ :

$l(i, j):=$ piecewise $(i=n$ and $j=n, a, i=n-1$ and $j=n, c, i=n-2$ and $j=n, d, i=n-3$ and $j=n, 0, i=n$ and $j=n-, c, i=n$ and $j=n-2, d, i=n$ and $j=n-3,0, h(i, j))$ :

for $i$ from 1 to 7 do

$W[i]:=\operatorname{Determinant}(\operatorname{Matrix}(h, i))$

end do:

for $i$ from 1 to 6 do

$n:=i: F[i]:=\operatorname{Determinant}(\operatorname{Matrix}(p, i))$

end do:

for $i$ from 1 to 5 do

$n:=i: G[i]:=$ Determinant $(\operatorname{Matrix}(l, i))$

end do: 
Step 2 (generating coefficients of equations)

$$
\begin{aligned}
& \alpha_{1}:=a: \alpha_{2}:=b d\left(b d-2 c^{2}\right): \alpha_{3}:=d^{2}\left(2 c^{3}-4 b c d+b^{2} c+a d^{2}\right): \\
& \alpha_{4}:=d^{3}\left(2 c^{2} d+b^{2} d-b c^{2}-d^{3}\right): \alpha_{5}:=-b c d^{5}: \alpha_{6}:=-b: \alpha_{7}:=b c: \\
& \alpha_{8}:=d\left(2 a c-b^{2}\right): \quad \alpha_{9}:=b d^{2}(2 c-a): \alpha_{10}:=d^{3}\left(2 b d-b^{2}-2 c^{2}\right): \\
& \alpha_{11}:=c d^{4}(b-2 d): \quad \alpha_{12}:=b d^{6}: \alpha_{13}:=-c^{2}: \alpha_{14}:=d(b c-d a): \\
& \beta_{1}:=b: \quad \beta_{2}:=-b c d^{2}: \quad \beta_{3}:=d^{3}\left(c^{2}-b d\right): \beta_{4}:=d^{5} c: \beta_{5}:=-c: \\
& \beta_{6}:=b d: \quad \beta_{7}:=a d^{2}: \quad \beta_{8}:=b d^{3}: \beta_{9}:=-c d^{4}: \beta_{10}:=-d^{6}: \beta_{11}:=-c d: \\
& \xi_{1}:=a: \quad \xi_{2}:=-c^{2}: \xi_{3}:=2 c d: \xi_{4}:=-d^{2}:
\end{aligned}
$$

Step 3 (assigning fixed data to matrix elements)

$a:=1: \quad b:=2: c:=3: d:=4$ :

Step 4 (main loop for determinant $W_{n}$ )

$$
\begin{aligned}
& \text { for } n \text { from } 1 \text { to } 99993 \text { do } \\
& W[n+7]:=\operatorname{evalf}\left(\alpha_{1} W[n+6]+\alpha_{2} W[n+3]+\alpha_{3} W[n+2]+\alpha_{4} W[n+1]+\alpha_{5} W[n]+\right. \\
& +\alpha_{6} F[n+6]+\alpha_{7} F[n+5]+\alpha_{8} F[n+4]+\alpha_{9} F[n+3]+\alpha_{10} F[n+2]+\alpha_{11} F[n+1]+ \\
& \left.+\alpha_{12} F[n]+\alpha_{13} G[n+5]+\alpha_{14} G[n+4]\right): \\
& F[n+6]:=\operatorname{evalf}\left(\beta_{1} W[n+5]+\beta_{2} W[n+2]+\beta_{3} W[n+1]+\beta_{4} W[n]+\beta_{5} F[n+5]+\right. \\
& \left.+\beta_{6} F[n+4]+\beta_{7} F[n+3]+\beta_{8} F[n+2]+\beta_{9} F[n+1]+\beta_{10} F[n]+\beta_{11} G[n+4]\right): \\
& G[n+5]:=\operatorname{evalf}\left(\xi_{1} W[n+4]+\xi_{2} W[n+3]+\xi_{3} F[n+3]+\xi_{4} G[n+3]\right):
\end{aligned}
$$

end do:

$\operatorname{print}(W[100000])$

At the end we get $-8.768970745 \cdot 10^{61547}$ as the value of determinant of the matrix under considerations. It can be emphasized that all calculations are performed with Maple default precision (Digits $=10$ ).

\section{Conclusions}

It was shown that the determinant of the heptadiagonal symmetric Toeplitz matrix can be obtained as a particular solution of the system of three homogeneous linear recurrence equations. Moreover, it was presented that in the case of determinant of pentadiagonal symmetric Toeplitz matrix the above system reduces into two linear recurrence equations. Whilst in the case of determinant of pertinent 
tridiagonal matrix we finally obtain one equation. The general considerations are illustrated by the example in which the implementation of the proposed approach to the Maple system was presented.

\section{References}

[1] Elouafi M., A note for an explicit formula for the determinant of pentadiagonal and heptadiagonal symmetric Toeplitz matrices, Applied Mathematics and Computation 2013, 219, 4789-4791.

[2] Elouafi M., On a relationship between Chebyshev polynomials and Toeplitz determinants, Applied Mathematics and Computation 2014, 229, 27-33.

[3] Solary M.S., Finding eigenvalues for heptadiagonal symmetric Toeplitz matrices, J. Math. Anal. Appl. 2013, 402, 719-730.

[4] Borowska J., Łacińska L., Rychlewska J., A system of linear recurrence equations for determinant of pentadiagonal matrix, Journal of Applied Mathematics and Computational Mechanics 2014, 13(2), 5-12.

[5] Elaydi S., An Introduction to Difference Equations, Springer, 2005. 